\title{
Managing patients taking tumour necrosis factor inhibitors
}

\author{
Tim Y-T. Lu, Registrar, and Catherine Hill, Staff specialist, Department of Rheumatology, \\ Queen Elizabeth Hospital, Adelaide
}

\section{Summary}

Patients with rheumatoid arthritis, ankylosing spondylitis, psoriatic arthritis or juvenile chronic arthritis that is unresponsive to standard diseasemodifying antirheumatic drugs can now be treated with tumour necrosis factor inhibitors. These biological drugs all antagonise the actions of tumour necrosis factor, a key cytokine central to the inflammatory cascade. Their adverse effects can be severe and include sepsis, reactivation of pulmonary tuberculosis, blood dyscrasias, demyelinating syndromes, lymphoproliferative disease and precipitation of cardiac failure. Careful monitoring of patients is important.

Key words: adalimumab, etanercept, infliximab, rheumatology.

(Aust Prescr 2006;29:67-70)

\section{Introduction}

In recent years the treatment of inflammatory joint diseases has undergone a dramatic change with the advent of biological drugs. Patients with rheumatoid arthritis, ankylosing spondylitis, psoriatic arthritis and juvenile chronic arthritis are potential candidates for this new therapy. Biological drugs are usually prescribed if the patient's condition has been resistant to treatment with standard disease-modifying antirheumatic drugs or if the patient has been unable to tolerate them. ${ }^{1}$ In clinical trials biological drugs have been superior to disease-modifying antirheumatic drugs in achieving disease remission and retarding bony destruction. The most common biological drugs used today belong to the class known as tumour necrosis factor (TNF) inhibitors. This class includes infliximab, adalimumab and etanercept. As these drugs are being used more frequently, it is important to be familiar with the principles of managing patients during treatment in the community.

\section{Mechanism of action}

Tumour necrosis factor is a critical pro-inflammatory cytokine in the cascade of inflammatory joint diseases. ${ }^{2}$ It mediates many of the inflammatory processes in rheumatoid arthritis including immune-cell activation and proliferation, apoptosis and regulation of leucocyte movement. TNF inhibitors act to neutralise the actions of this cytokine by binding to TNF or its receptor. Infliximab and adalimumab are recombinant monoclonal antibodies while etanercept is a soluble TNF-receptor fusion protein.

In randomised double-blind placebo-controlled trials TNF inhibitors have significantly improved the clinical and radiological outcomes for patients whose rheumatoid arthritis has been poorly controlled by standard disease-modifying antirheumatic drugs. The majority of trials involved combination therapy with methotrexate.

\section{Administration of TNF inhibitors}

Infliximab is given by intravenous infusion while adalimumab and etanercept are self-administered as subcutaneous injections (Table 1). For rheumatoid arthritis infliximab is commenced at $3 \mathrm{mg} / \mathrm{kg}$ of body weight at 0,2 and 6 weeks, then every 8 weeks. (In patients with an incomplete response, the maintenance dose may be gradually increased to a maximum of $10 \mathrm{mg} / \mathrm{kg}$.) The dose of adalimumab is $\mathbf{4 0 ~} \mathrm{mg}$ fortnightly, while etanercept is given at $25 \mathrm{mg}$ twice weekly or $50 \mathrm{mg}$ once a week.

\section{Table 1}

Dose schedule for TNF inhibitors

\begin{tabular}{llll}
\hline Drug & Presentation & Route of administration & Dose in rheumatoid arthritis \\
\hline Infliximab & $\begin{array}{l}\text { Powder for } \\
\text { reconstitution }\end{array}$ & Intravenous infusion & $\begin{array}{l}3 \mathrm{mg} / \mathrm{kg} \text { repeated after 2 weeks and 6 weeks, } \\
\text { then every } 8 \text { weeks }\end{array}$ \\
Adalimumab & Pre-filled syringe & Subcutaneous injection & $40 \mathrm{mg}$ repeated every 2 weeks \\
Etanercept & $\begin{array}{l}\text { Powder for } \\
\text { reconstitution }\end{array}$ & Subcutaneous injection & $25 \mathrm{mg}$ twice a week, or 50 mg once a week \\
\hline
\end{tabular}

TNF tumour necrosis factor 


\section{Indications}

TNF inhibitors are indicated for rheumatoid arthritis, ankylosing spondylitis, psoriatic arthritis and juvenile chronic arthritis, however they are not subsidised by the Pharmaceutical Benefits Scheme for all these indications. The prescription of TNF inhibitors by a rheumatologist or clinical immunologist for adult inflammatory joint diseases must follow strict guidelines as set out in the Schedule of Pharmaceutical Benefits. These guidelines are under constant review and are updated to reflect results from new clinical trials. Psoriatic arthritis, for example, is an indication currently under review. As of June 2005 all TNF inhibitors are indicated for rheumatoid arthritis irrespective of the patient's rheumatoid factor status. In addition, infliximab and etanercept are indicated for ankylosing spondylitis. Only etanercept has been approved for juvenile chronic arthritis.

The efficacy ofTNF inhibitors on the signs, symptoms, function, quality of life and retardation of radiological progression in patients with rheumatoid arthritis has been shown in a variety of roles. These include monotherapy, combination with methotrexate and in patients who have not previously been treated or have failed to respond to disease-modifying drugs. Combining aTNF inhibitor, especially infliximab, with methotrexate increases efficacy.

As there have been no direct head-to-head trials, no singleTNF inhibitor can be said to be more effective than another. The choice of drug therefore depends on the patient's preference and the prescriber's experience with the particularTNF inhibitor. This includes considering the contraindications (see Box 1).

\section{Before treatment}

As part of the pre-treatment assessment, patients should have a chest X-ray to exclude latent pulmonary tuberculosis and congestive cardiac failure. The full blood count, liver function tests, hepatitis $B$ and $C$ serology, inflammatory markers

\section{Box 1}

\section{Contraindications to TNF inhibitors ${ }^{11}$}

Previous untreated tuberculosis

Recurrent chest infections/bronchiectasis

Septic arthritis within 12 months

Infected prosthesis

Indwelling urinary catheter

Multiple sclerosis/demyelinating illness

Malignancy within 10 years (apart from fully resected basal cell carcinoma more than five years before)

Pregnancy and lactation

Congestive heart failure

Chronic cutaneous ulceration (but not pyoderma gangrenosum)
(CRP, ESR), and anti-nuclear and anti-double-stranded DNA autoantibodies should be checked before therapy. The presence of latent pulmonary tuberculosis warrants eradication therapy, usually for nine months, before starting aTNF inhibitor. This is best managed by a physician experienced in tuberculosis care.

\section{Adverse effects}

Given that TNF inhibitors are relatively new drugs, their long-term safety is still being established. From their mechanism of action it is predicted that there will be an increase in the incidence of infection and possibly secondary malignancy.

The pharmacovigilance database of the Australian Rheumatology Association contains many case reports of toxicities related toTNF inhibitors. These include severe injection-site reactions, infection (particularly mycobacterial and opportunistic organisms), lymphoproliferative disorders, lupus-like autoimmune disease, demyelinating disease, haematological abnormalities including aplastic anaemia, and precipitation of cardiac failure. These findings are important as infection (particularly pulmonary infection), cardiovascular disease and osteoporosis are the three conditions that cause the greatest morbidity and mortality in rheumatoid arthritis. ${ }^{3}$

\section{Injection-site reactions}

In patients receiving adalimumab and etanercept, injection-site reactions present as mild erythema, itching, pain or swelling, usually lasting a few days. It is important that patients mix etanercept correctly before injecting it. During infusion of infliximab some patients complain of headache and nausea. An immediate hypersensitivity response suggested by the development of rash, urticaria or anaphylaxis is rare. Patients rarely cease therapy because of injection-site reactions.

\section{Infection}

Many infections can occur. These include serious bacterial infections, tuberculosis, atypical mycobacterial infection, aspergillosis, histoplasmosis, listeriosis, Pneumocystis jiroveci pneumonia and cryptococcal infections. Infections may be more common in patients over 65 years of age than in younger patients. ${ }^{4}$

Latent viral infections may be reactivated. These include herpes simplex virus (including genital herpes), herpes zoster virus and cytomegalovirus.

Reactivation of tuberculosis has been reported in association with allTNF inhibitors. This usually occurs within the first 2-5 months of commencing treatment. The majority of cases present as extra-pulmonary and disseminated tuberculosis. A recent study has shown that screening for previous pulmonary tuberculosis with chest $\mathrm{X}$-ray and Mantoux testing followed by appropriate treatment before starting TNF inhibitors, significantly reduces the incidence of tuberculosis. ${ }^{5}$ 


\section{Lymphoproliferative disease}

Cases of lymphoma have been reported in patients taking TNF inhibitors. The incidence of lymphoma and leukaemia is already increased in patients with rheumatoid arthritis, particularly those with high disease activity. Epidemiological studies differ on whether there is an additional risk of lymphoproliferative disorders among patients with rheumatoid arthritis who are treated with TNF inhibitors. ${ }^{6,7}$ There is no indication that patients with rheumatoid arthritis taking TNF inhibitors are at increased risk of other tumours.

\section{Blood dyscrasias}

Medically significant thrombocytopenia and leucopaenia have been reported with TNF inhibitors. However, pancytopaenia including aplastic anaemia rarely occurs. These abnormalities are generally reversible upon cessation of the drug.

\section{Lupus-like autoimmune responses}

Positive antinuclear antibodies develop in over $50 \%$ of patients with rheumatoid arthritis during treatment with infliximab and $13 \%$ develop antibodies to double-stranded DNA. ${ }^{8}$ With etanercept, $11 \%$ of patients develop a new antinuclear antibody and $2 \%$ develop double-stranded DNA antibodies. ${ }^{9}$ Despite the high rate of autoantibodies, clinical manifestations of drug-induced systemic lupus erythematosis are rare.

\section{Demyelinating syndromes}

Exacerbations of previously quiescent multiple sclerosis and the onset of other demyelinating diseases (such as optic neuritis) have been reported in patients taking TNF inhibitors. Symptoms included paraesthesia, visual disturbance and confusion.

\section{Cardiac failure}

Patients with heart failure have elevated concentrations ofTNF. Trials of etanercept and infliximab in heart failure were stopped early because there was no evidence that TNF inhibitors were of benefit. In the case of infliximab, mortality increased. ${ }^{10}$ TNF inhibitors are therefore contraindicated in patients with heart failure (New York Heart Association class III and IV).

\section{Pregnancy and lactation}

There are no clinical data for pregnant or lactating women being treated with TNF inhibitors. Animal studies were inconclusive with regard to the embryofetal toxicity so their use during pregnancy is not recommended. Women of childbearing age should be advised to use effective contraception during therapy.

\section{Practice points (Box 2)}

Before starting a patient onTNF inhibitors, immunisations should be brought up to date. Live vaccines should not be given to people receiving TNF inhibitors. There is no contraindication to yearly influenza and five-yearly pneumococcal vaccination. Varicella vaccination should be considered for individuals who are seronegative. Routine screening for latent tuberculosis is mandatory before treatment. It is also important to remain clinically vigilant for reactivated tuberculosis, as a delayed diagnosis may lead to increased morbidity. Patients are reassessed after 8-12 weeks of treatment to see if they qualify for continuation of the drug. Routine clinical examination for signs of cardiac failure and pulmonary sepsis should be performed at each visit. Measurement of the full blood count at baseline and at regular intervals (initially monthly then three-monthly thereafter) is needed to monitor for blood dyscrasias. This may differ if patients are on other therapy such as methotrexate or leflunomide.

Cold application, simple analgesic drugs such as paracetamol, and antihistamines are adequate for treating minor injection-site reactions in patients administering adalimumab and etanercept. Rotation of injection sites is also a useful strategy to prevent skin irritation. Slowing the rate of infusion may ameliorate the infusion-related adverse reactions to infliximab.

The development of systemic or localised infection warrants cessation or postponement of TNF inhibitor therapy. Treatment can be continued after the infection has resolved. For patients exposed to chickenpox or shingles during therapy their serological status should be obtained. Those with a negative serology will require treatment with zoster immunoglobulin to prevent disseminated infection. For patients undergoing major surgery, it is prudent to interruptTNF inhibitor treatment until the risk of postoperative infection has declined. Minor surgery such as dental procedures does not require cessation of therapy. When patients develop serious complications during treatment, the TNF inhibitors are to be stopped. Specialist advice should be sought immediately.

\section{Box 2 \\ Practice points}

Update immunisations, including yearly influenza vaccines - avoid live vaccines during treatment

Clinical examination for signs of cardiac failure and pulmonary sepsis

Monthly complete blood count, renal and liver function tests if on methotrexate, C-reactive protein and erythrocyte sedimentation rate

Monitor for drug toxicities

Withhold treatment if:

- hypersensitivity

- active sepsis

- malignancy

- pregnancy or lactation

- worsening congestive cardiac failure

- surgery 


\section{References}

1. Lee ATY, Pile K. Disease modifying drugs in adult rheumatoid arthritis. Aust Prescr 2003;26:36-40.

2. McColl G. Tumour necrosis factor alpha inhibitors for the treatment of adult rheumatoid arthritis. Aust Prescr 2004;27:43-6.

3. O'Dell JR. Therapeutic strategies for rheumatoid arthritis. N Engl J Med 2004;350:2591-602.

4. Olsen NJ, Stein CM. New drugs for rheumatoid arthritis. N Engl J Med 2004;350:2167-79.

5. Carmona L, Gómez-Reino JJ, Rodríguez-Valverde V, Montero D, Pascual-Gómez E, Mola EM, et al. Effectiveness of recommendations to prevent reactivation of latent tuberculosis infection in patients treated with tumor necrosis factor antagonists. Arthritis Rheum 2005;52:1766-72.

6. Askling J, Fored CM, Baecklund E, Brandt L, Backlin C, Ekbom A, et al. Haematopoietic malignancies in rheumatoid arthritis: lymphoma risk and characteristics after exposure to tumour necrosis factor antagonists. Ann Rheum Dis 2005;64:1414-20.

7. Geborek P, Bladstrom A, Turesson C, Gulfe A, Petersson IF, SaxneT, et al. Tumour necrosis factor blockers do not increase overall tumour risk in patients with rheumatoid arthritis, but may be associated with an increased risk of lymphomas. Ann Rheum Dis 2005;64:699-703.
8. Lipsky PE, van der Heijde DM, St Clair EW, Furst DE, Breedveld FC, Kalden JR, et al. Infliximab and methotrexate in the treatment of rheumatoid arthritis. $\mathrm{N}$ Engl J Med 2000;343:1594-602.

9. Roberts L, McColl GJ. Tumour necrosis factor inhibitors: risks and benefits in patients with rheumatoid arthritis. Int Med J 2004;34:687-93.

10. Kwon HJ, CoteTR, Cuffe MS, Kramer JM, Braun MM. Case reports of heart failure after therapy with a tumor necrosis factor antagonist. Ann Intern Med 2003;138:807-11.

11. Nash P, Florin TH. Tumour necrosis factor inhibitors. Med J Aust 2005;183:205-8.

Conflict of interest: none declared

\section{Self-test questions}

The following statements are either true or false (answers on page 87)

3. Tumour necrosis factor inhibitors can reactivate tuberculosis.

4. Influenza vaccination is contraindicated during treatment with tumour necrosis factor inhibitors.

\section{Book review}

Pocket guide to lung function tests: explanations without equations. $2^{\text {nd }}$ edition. Hancox B, Whyte K.

\section{Sydney: McGraw-Hill; 2006. 176 pages. Price $\$ 34.95$}

\section{M.C.F. Pain, Honorary consultant physician, Department of Respiratory Medicine, The Royal Melbourne Hospital, Melbourne}

This little book first appeared in 2001 with a reprint in 2003. The appearance of a second edition shows that it has found a market. Although not specifically spelt out, it appears that the second edition has been 'revised throughout' with the addition of chapters 12 and 13 dealing with the preoperative assessment for thoracic surgery and fitness for flying. The basic approach in the interpretation of tests is of pattern recognition rather than requiring much knowledge of pure respiratory physiology and I have no quarrel with that. Most of us read an ECG without much knowledge of cardiac electrophysiology.

In nine chapters covering the commonly used tests, from spirometry to cardiopulmonary exercise tests, the authors present a commonsense approach in a compact yet highly readable text laced with clear diagrams and clinical examples. Things become a little more esoteric and perhaps premature in chapter 10 (exhaled nitric oxide) and some 'non-routine tests' are discussed in chapter 11 .

With so much useful information packed into a small book (the pages are $18 \times 11.5 \mathrm{~cm}$ ) it is hard to be critical, but a few minor things caught my eye. There is inconsistency in the use of the symbols so beloved by respiratory physiologists (for example VD in the glossary and $V_{D}$ in the text). Despite stating that the book uses $\mathrm{mmHg}$ rather than $\mathrm{kPa}$ units, chapter 13 uses $\mathrm{kPa}$. Mixed spirometric patterns would be better introduced in chapter 1 rather than chapter 4 . A pitfall not mentioned with oximetry is the dependence on adequate circulation. The delay in elevation in carbon dioxide tension in ventilation/perfusion mismatching has less to do with solubility and more to do with the complexity of the carbon dioxide-oxygen-haemoglobin relationships in hypoxia, and even multi-breath nitrogen clearance will only be linear if plotted semi-logarithmically.

The book can be read slowly in 90 minutes and this would be time well spent by candidates for postgraduate exams, advanced trainees in general and thoracic medicine, respiratory scientists and nurses. There are good references for further study and an excellent index. Whether it will be carried around in many pockets is another matter, but it is a compact reference which deserves a wide readership. 